\title{
Comparative numerical study on pressure drop in helically coiled and longitudinally C-shaped pipes
}

\author{
Yassine Demagh $^{1}$ (D) Elwardi Bitam ${ }^{1} \cdot$ Lyes Bordja $^{2}$
}

Received: 15 October 2019 / Accepted: 17 August 2020 / Published online: 27 August 2020

(c) Springer Nature Switzerland AG 2020

\begin{abstract}
In the present work, the shear stress transport (SST) $k-\omega$ model is used to simulate internal turbulent flows in curved tubular heat exchangers and the resulting friction factors $C_{f}$ are calculated. A helically coiled configuration was used in the validation process of the model that showed a good agreement between numerical results of the friction factors $C_{f}$ and the standard experimental correlations of the literature, with a maximum relative error of $4.24 \%$. A comparative study was performed to investigate the possibility of applying friction factor correlations, established mainly for helical coils, to longitudinally $C$-shaped pipes. Preliminary results confirm the inability of coil-specific correlations to predict the friction factor $C_{f}$ inside $C$-shaped pipes. It was also noted that, unlike helical coils, the $C$-shaped pipe friction factor appears to be affected by ratios other than the curvature ratio as highlighted in this study by introducing the effect of the amplitude ratio $\beta$ defined as the ratio of the amplitude to the curvature radius.
\end{abstract}

Keywords Pressure drop · Fanning friction factor $\left(C_{f}\right) \cdot$ Helically coiled pipes $\cdot$ Longitudinally C-shaped pipes

\section{List of symbols}

A Amplitude of the C-shaped pipe (m)

$C_{f} \quad$ Friction coefficient

$c_{p} \quad$ Heat capacity $\left(\mathrm{J} \mathrm{kg}^{-1} \mathrm{~K}^{-1}\right)$

$D_{i} \quad$ Inner diameter of the pipe $(\mathrm{m})$

$f \quad$ Darcy friction factor

$\mathrm{Nu}$ Nusselt number

Pr Prandlt number

$\operatorname{Pr}_{t} \quad$ Turbulent Prandtl number

$R_{c} \quad$ Curvature radius (m)

Re Reynolds number

$\mathrm{T}$ Temperature (K)

$u \quad$ Velocity field $\left(\mathrm{m} \mathrm{s}^{-1}\right)$

\section{Greek letters}

$\beta \quad$ Amplitude ratio

$\delta \quad$ Curvature ratio

$\lambda$ Periodicity length ( $\mathrm{m})$. Heat conductivity $\left(\mathrm{W} \mathrm{K}^{-1} \mathrm{~m}^{-2}\right.$ )

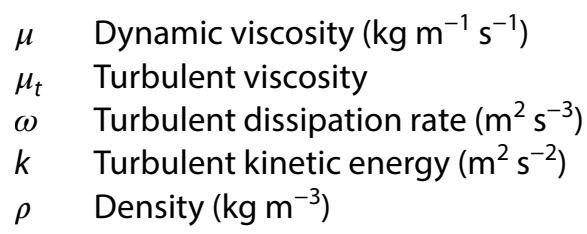

\begin{tabular}{ll}
\multicolumn{2}{c}{ Subscripts } \\
b & Bulk \\
f & Fluid \\
i & Inner \\
in & Inlet \\
s & Straight \\
w & Wall
\end{tabular}

$\checkmark$ Yassine Demagh, y.demagh@univ-batna2.dz; Elwardi Bitam, bitam.ew@gmail.com; Lyes Bordja, lyes_b2003@yahoo.fr| ${ }^{1}$ LESEI, Department of Mechanics, University Batna 2, Batna, Algeria. ${ }^{2}$ Department of Mechanics, Faculty of Science and Applied Sciences, University Larbi Ben M’hidi, Oum El Bouaghi, Algeria. 


\section{Introduction}

Due to improved heat transfer rate, the most widely used tubular heat exchangers in industrial processes are the helically coiled pipes (Fig. 1a). Nevertheless, they produce significant and undesirable pressure drop because of the generation of second flows (vortices) within the mainstream. In fact, concern about pressure drop in curved configurations is common, with particular attention to the helical coils (Hart et al. [1], Guo et al. [2] and Mori and Nakayama [3]). For most cases, the curvature ratio $\delta=D_{i} / 2 R_{c}$ (pipe inner diameter to twice the curvature radius) is considered as the relevant parameter to characterise the pressure drop. For more detailed explanations and observations on the subject the reader is referred to the review paper by Vashisth et al. [4].

Besides helical coils, longitudinally curved pipes (C-shaped, sinusoidal...) have been relatively less studied due to limited use in industry, which had led to a lack of correlations predicting turbulent heat transfer or pressure drop within it. Recently, Demagh et al. $[5,6]$ and Bitam et al. [7] highlighted the concept of replacing straight absorbers in linear concentrating solar power (CSP) collectors by wavy ones; this last application motivated the present study.

(a)

(b)
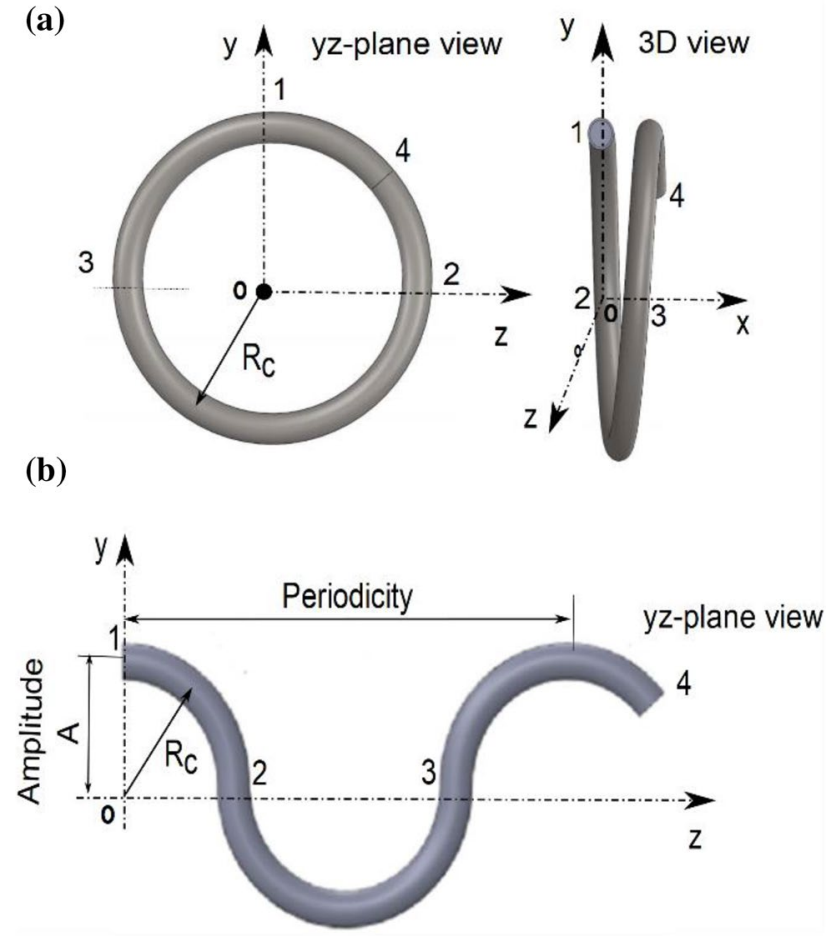

Fig. 1 Studied configurations. a The helically coiled pipe. b The longitudinally C-shaped pipe

SN Applied Sciences

A SPRINGER NATURE journal
In this paper, a comparative study on the pressure drop in helically coiled and longitudinally C-shaped pipes was conducted to examine the possible use of Fanning friction factor $\left(C_{f}\right)$ correlations developed for the helically coiled pipes to C-shaped pipes. A CFD-model (Fluent Inc.), based on the (SST) $k-\omega$ turbulent model, was used to simulate conjugated heat transfer and dynamic behaviour of internal flows of water through curved pipes.

\section{C-shaped pipe versus helically curved pipe}

The helically coiled pipe (Fig. 1a) is defined by sweeping a circular section along its helical centreline, and is fully characterized by the curvature radius $R_{c}(=136.67 \mathrm{~mm})$, and the inner diameter of the pipe $D_{i}(=8 \mathrm{~mm})$. The curvilinear length is about $976.11 \mathrm{~mm}$.

By fixing, the coil at the two points (pivots) 2 and 3 (Fig. 1a) then turning its free ends (branches (1-2) and (4-3)) $180^{\circ}$ around the $y$ axis, one gets the $C$-shaped configuration in Fig. 1 b.

\section{Problem formulation}

The realizable $k-\varepsilon$ two-equation model is among the most used turbulent models to study the fluid turbulent flow through helical pipes (Li et al. [8], Jayakumar et al. [9], Yang et al. [10], and Pawar et al. [11]). Di Piazza and Ciofalo [12] discussed the use of different turbulence models for simulating internal flows in curved pipes, including the standard $k-\varepsilon$ model with wall functions, the (SST) $k-\omega$ model and a second order Reynolds Stress- $\omega$ model. It has been established that the (SST) $k-\omega$ model by Menter [13] gives the best agreement with experimental data. In this study, the steady state, incompressible and turbulent (SST) $k-\omega$ CFD-model, developed previously to give highly accurate predictions of the amount of flow separation under adverse pressure gradients [13], is used for all simulations. The model includes the general governing equations of the problem, written in the Cartesian tensor notation as:

The mass conservation equation $[7,13]$,

$\frac{\partial u_{i}}{\partial x_{i}}=0$

The momentum conservation equations [7, 13],

$\frac{\partial}{\partial x_{j}}\left(\rho u_{i} u_{j}\right)=-\frac{\partial p}{\partial x_{i}}+\frac{\partial}{\partial x_{j}}\left(\left(\mu+\mu_{t}\right)\left(\left(\frac{\partial u_{i}}{\partial x_{j}}+\frac{\partial u_{j}}{\partial x_{i}}\right)-\frac{2}{3} \frac{\partial u_{k}}{\partial x_{k}}\right)\right)$

The energy conservation equation $[7,13]$, 
$\frac{\partial}{\partial x_{j}}\left(\rho u_{i} c_{p} T\right)=\frac{\partial}{\partial x_{i}}\left(\left(\lambda+\frac{c_{p} \mu_{t}}{\operatorname{Pr}_{t}}\right) \frac{\partial T}{\partial x_{i}}\right)$

$u, \lambda, \mu, \mu_{t} \rho$ and $c_{p}$ being, respectively, the velocity field, thermal conductivity, dynamic viscosity, turbulent viscosity, density, and heat capacity. The turbulent Prandtl number $\mathrm{Pr}_{t}$ is set at 0.85 .

For the sake of brevity, the equations of the turbulent kinetic energy $(k)$ and the dissipation rate $(\omega)$ will not be presented in this paper; for more details, please refer to [13].

All simulations are carried out as steady state solutions of the Navier-Stokes equations, energy equation and turbulence equations. To increase the accuracy of the numerical results and reduce the numerical diffusion, second order upwind discretization scheme is used for pressure, momentum, energy, $(k)$ and $(\omega)$. The standard SIMPLE scheme is used for the pressure-velocity linking.

The convergence criteria were set at $10^{-4}$ for all variables except for the energy equation where the value of $10^{-6}$ was adopted. The fluid properties are temperature depending, and the boundary conditions are as follows:

- A mass inlet boundary condition: $\dot{m}=\dot{m}_{i n}$ at $T_{\text {in }}=310^{\circ} \mathrm{C}$,

- Fully-developed condition at the outlet,

- Adiabatic boundary conditions for solid ends of the pipes,

- The outer surface of the pipes is set at a constant temperature of $315^{\circ} \mathrm{C}$,

- For the C-shaped pipes, the symmetry condition with regard to the yz-plane was adopted.
Table 1 Grid independency checking

\begin{tabular}{lllll}
\hline Mesh & Cells & $\approx y^{+}$ & $f$ & $\% f$ \\
\hline 1 & $1,081,236$ & 2.59 & 0.005914771 & - \\
2 & $1,081,236$ & 1.32 & 0.005924864 & 0.17 \\
3 & $1,081,236$ & 0.26 & 0.005990722 & 1.28 \\
4 & $3,863,913$ & 0.054 & 0.005978305 & 1.07 \\
\hline
\end{tabular}

For the helically coiled pipe simulations are performed assuming the entire configuration (Fig. 2a, b). Whereas for the $\mathrm{C}$-shaped pipe only half was used taking into account the symmetry boundary condition in relation to yz-plane, as shown in Fig. 2c. The symmetry boundary condition is strongly related to the direction of the normal vector $(\vec{n})$ of bends with regard to the direction of the gravitational field vector $(\vec{g})$. If the directions are collinear (standing position of the pipe in Fig. 2c), symmetry could be assumed, otherwise (in the inclined or lying position) it should not be. Considering the symmetry condition of the C-shaped pipes will minimize, among others, the computational efforts for this case.

Also shown in Fig. 2, the structured mesh of the pipe wall obtained using quadrilateral elements, and the unstructured one for the fluid using quad/mixed elements. To satisfy the $y^{+}<1$ constraint of the (SST) $k-\omega$ model, a non-uniform high refinement of the viscous sub-layer is performed. The grid independence tests were executed to ensure the validity of the numerical results, sample of these tests is summarised in Table 1. The solution is considered mesh independent when the variation of the predicted average friction factor is close to $1 \%$. It is found that the results of the mesh 3 are not affected by

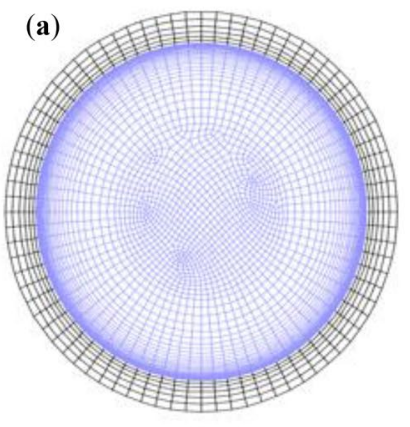

(b)
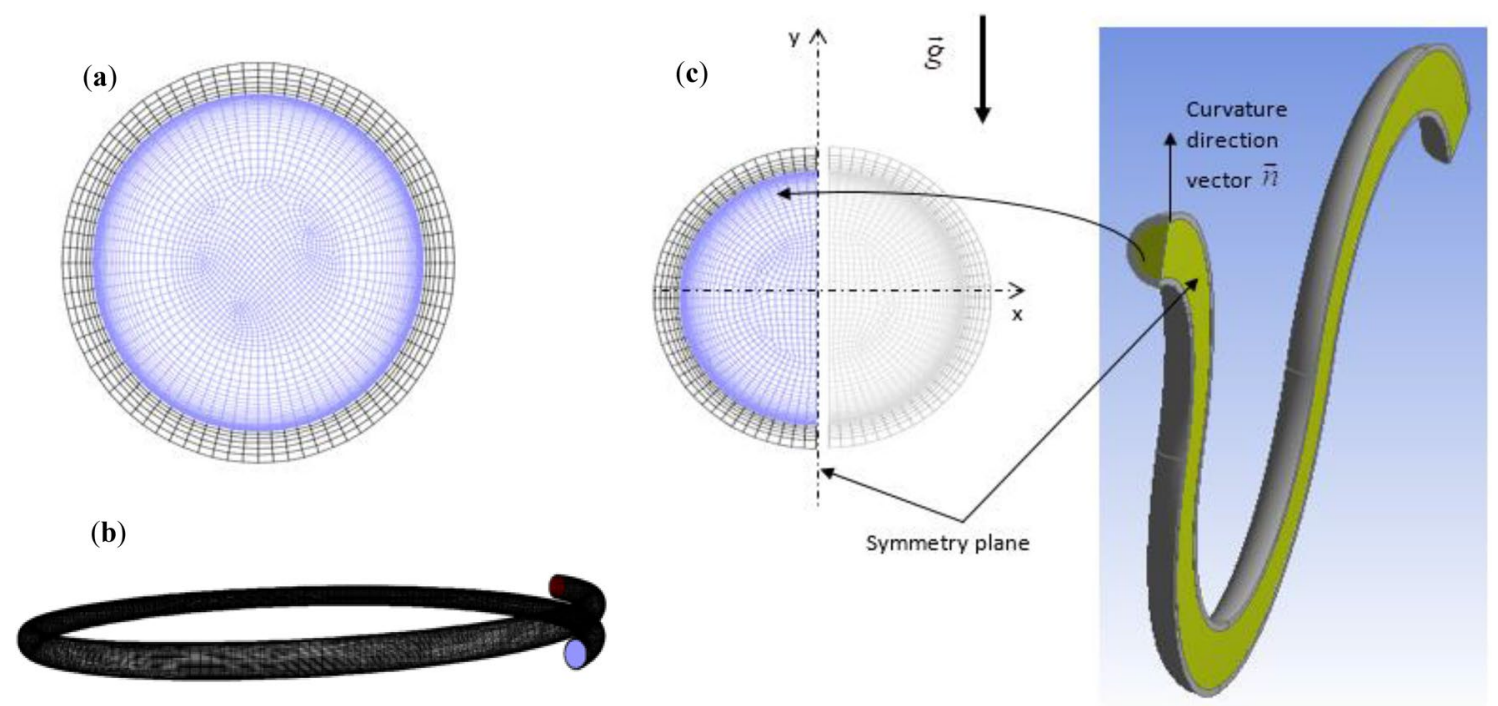

Fig. 2 Typical meshing for analysis. a Grid at cross-sections. b Helical coil meshing. c 3D view of longitudinally C-shaped pipe 
the refinement and therefore the mesh 3 is used in the present study.

\section{Results}

For the validation of the numerical results, the correlations of the Fanning friction factor $C_{f}$ are used. Not to confuse with the Darcy friction factor $f$, the two friction factors related as $C_{f}=f / 4[14,15]$.

$C_{f}$ was obtained through three different ways:

- Using correlation by Gnielinski [14] based on water flowing through a heated helically curved pipe, which can be presented as,

$$
C_{f}=\frac{1}{4}\left[0.3164 / R e^{0.25}+0.03 \cdot \delta^{0.5}\right]\left(\frac{\mu_{w}}{\mu_{b}}\right)^{0.27}
$$

$\mu_{b}$ and $\mu_{w}$ are the fluid viscosity defined respectively at the bulk temperature and the wall temperature. All properties of the fluid are estimated at the inlet bulk temperature of the water.

- Through the most popular correlation in curved pipes for fully turbulent flow by Ito [16], which can be defined as:

$$
C_{f}=\frac{1}{4}\left(0.304 R e^{-0.25}+0.029 \sqrt{\delta}\right)
$$

- Finally, through correlation by Abou-Arab et al. [17] for wavy pipes, as defined below:

$$
C_{f}=\frac{1}{4}\left(f_{s}+0.005 \delta^{0.5}\right)
$$

$f_{s}=(0.790 \operatorname{Ln}(\operatorname{Re})-1.64)^{-2}$ is the friction factor for smooth and straight tube by Petukhov [15].

Figure 3 shows variation of friction factor with Reynolds number for helically coiled configuration. The numerical results closely agree with the experimental correlation by Ito and Gnielinski, with max relative errors of $3.68 \%$ and $4.28 \%$, respectively. Based on these results, the model accurately captures phenomena within helically coiled pipes and will be used in the following for the C-shaped ones.

The comparison of the helical coil against C-shaped pipe is shown in Fig. 4. The trends of the two curves suggest that the friction factor of the helical coil is higher than the longitudinally C-shaped pipe; the more the flow regime increases the more the gap between curves increases.

In order to further investigate these notes, one introduce in the following paragraph new parameter

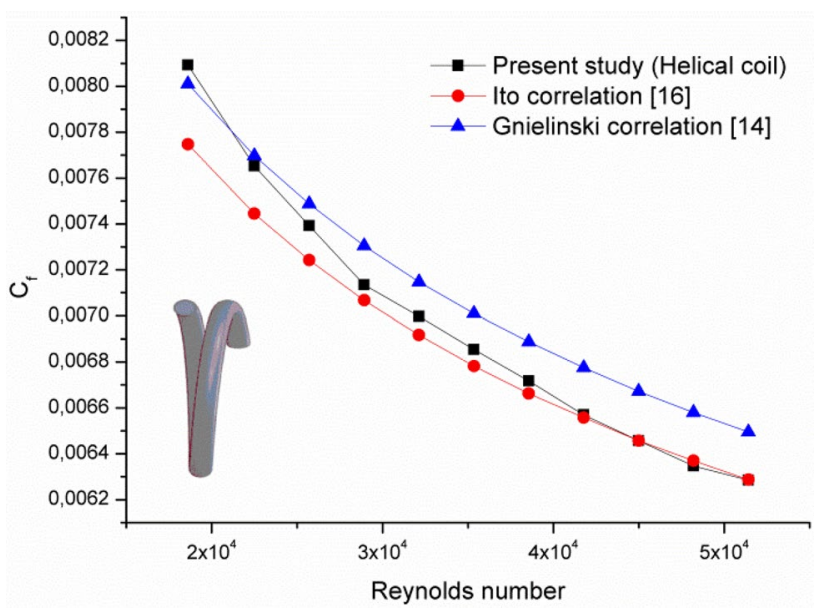

Fig. $3 C_{f}$ versus the flow regime Reynolds number: validation of the simulation results for the helically coiled pipe

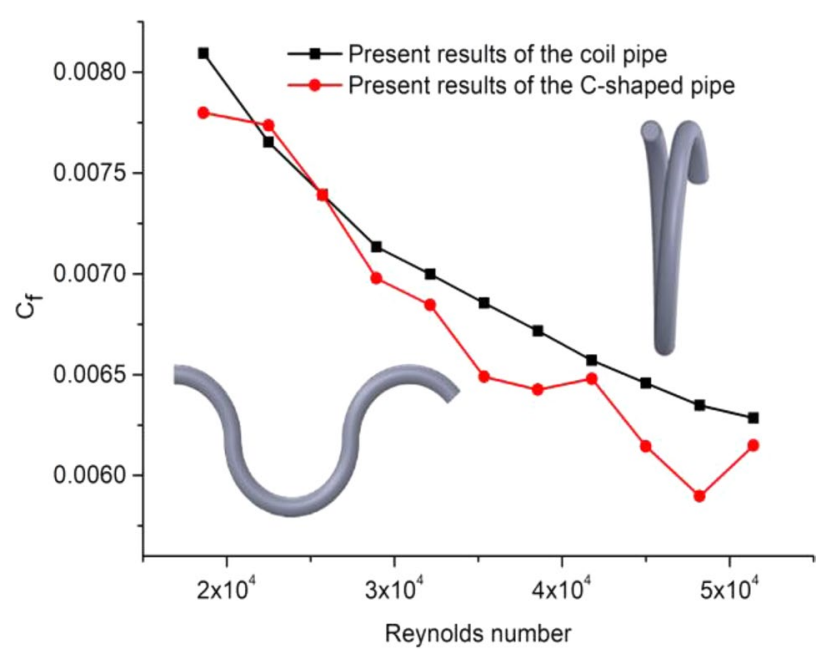

Fig. 4 Friction factors comparison between helical coil and C-shaped pipe

characterising the longitudinally curved pipes, named the amplitude ratio $\beta=A / R_{c^{\prime}}$ (ratio of the C-shaped pipe amplitude to the curvature radius).

As shown in Fig. 5, various C-shaped configurations could be obtained keeping the curvature ratio $\delta$ constant and varying the amplitude ratio $\beta$. Also, the periodicity, expressed as $\lambda=4 \sqrt{2 A \cdot R_{c}-A^{2}}$, is used to describe periodic geometries such $C$-shaped and sinusoidal pipes. In the present study, only the effect of the amplitude ratio on the friction factor will be considered.

It is known that the more the curvature ratio increases the more the friction factor increases too $[1-4,7,9,14$, $16-21]$, but what about the effect of the amplitude ratio 


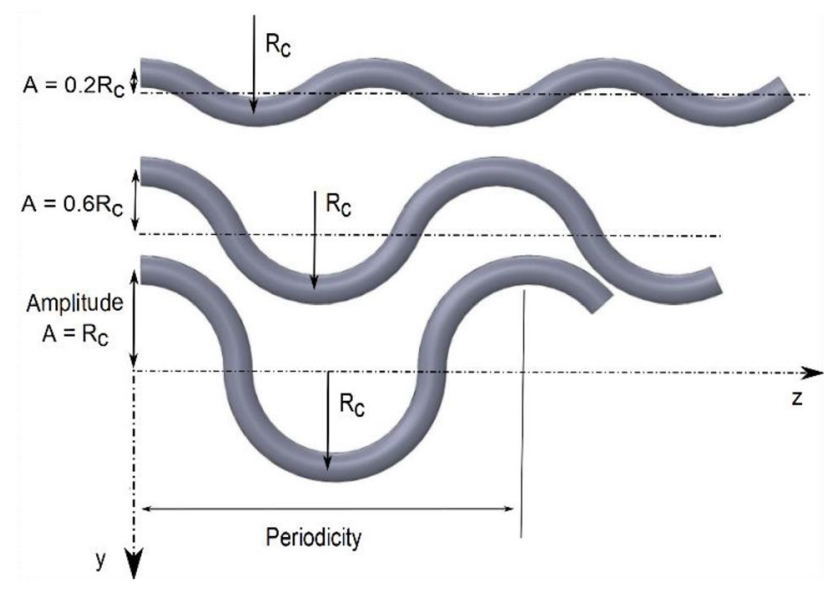

Fig. 5 C-shaped pipe configurations with various amplitude ratios $\beta$ and the same curvature ratio $\delta$

$\beta$ on $C_{f}$ keeping constant the curvature ratio and the inner diameter of the pipe?

Figure 6 answers this question and illustrates trends of obtained friction factors $C_{f}$ for $C$-shaped configurations varying $\beta$.

It is obvious that the friction factor $C_{f}$ changes; within the amplitude ratio range $0.2 \leq \beta \leq 1$, as $\beta$ decreases the friction factor decreases too.

Figure 7 illustrates the trends of the friction factor for various values of $\beta$ against correlations by Gnielinsky [14] and Ito [16].

The smaller $\beta$ the greater the gap between numerical results and correlations of $C_{f}$. With $\beta=1$, Fig. 7a showed that the friction factor reaches max relative errors of $11.59 \%$ and $8.02 \%$ against correlations of Gnielinski [14] and Ito [16], respectively. Whereas for the second configuration with $\beta=0.6$, the max relative error comparatively

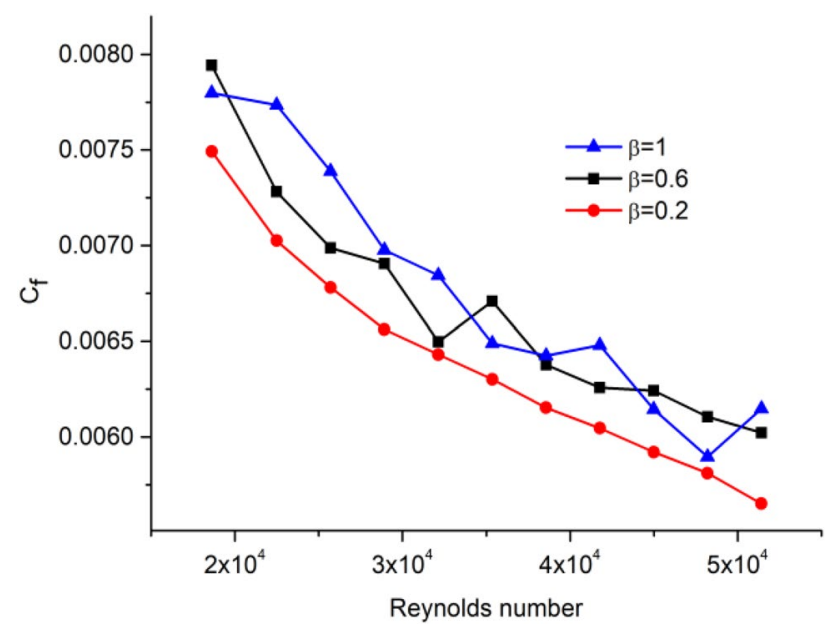

Fig. $6 C_{f}$ versus the Reynolds number for various amplitude ratio $\beta$

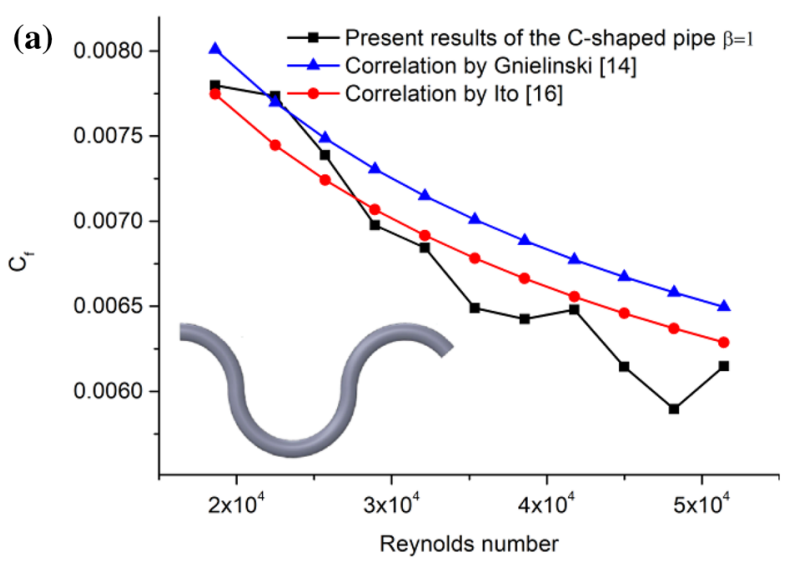

(b) $0.0080-\quad \rightarrow-$ Present results of the C-shaped pipe $\beta=0.6$
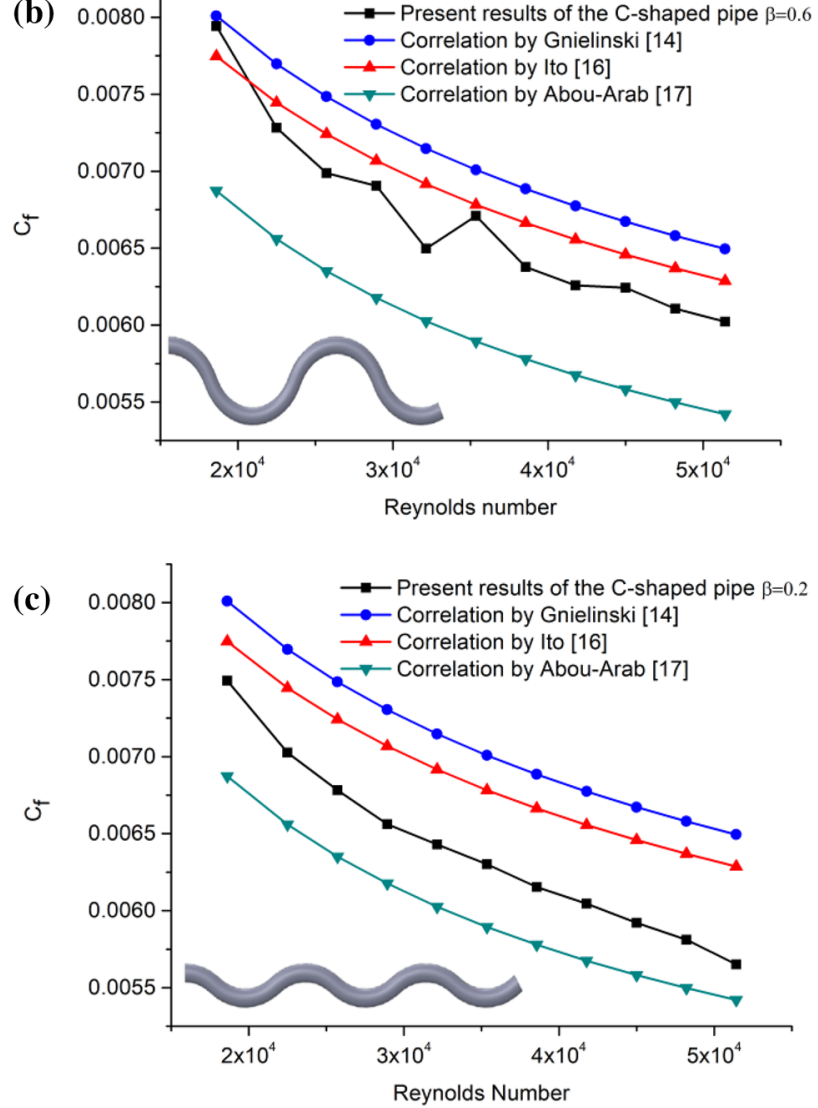

Fig. 7 Effects of $\beta$ on the C-shaped pipes friction factor. $\mathbf{a} \beta=1, \mathbf{b}$ $\beta=0.6$ and $\mathrm{c} \beta=0.2$

to Ito [16] was $6.46 \%$, as shown in Fig. $7 \mathrm{~b}$, and increases to $16 \%$ in Fig. $7 \mathrm{c}$ for the third configuration with $\beta=0.2$.

Unlike the correlation by Abou-Arab [17] (Eq. 6), which is developed for longitudinally sinusoidal pipes with $0.0111 \leq \delta \leq 0.71$, showed a max relative error of $9 \%$ for the configuration with $\beta=0.2$, and increases as $\beta$ increases giving a value of $13.47 \%$ for $\beta=0.6$. 
To explore the features of the flows through the pipes, the contours of velocity and temperature at various planes along both configurations were illustrated in Fig. 8 .

\section{Discussions}

As shown in Figs. 4, 6 and 7, it is obvious that the helically coiled and longitudinally C-shaped pipes do not have the same friction factors; the gap between results is considerable.

Also, $C_{f}$ of the C-shaped pipe appears to be affected by ratios other than the curvature ratio as highlighted in Figs. 6 and 7 which introduce the effect of the amplitude ratio $\beta$. As the amplitude ratio $\beta$ decreases the fiction factor decreases too.

In fact, this behaviour can be explained by the decreases of the periodic length $\lambda$ and the increases of the number of periods per length (Frequency $\approx 1 / \lambda$ ) as the amplitude ratio $\beta$ decreases, as shown in Fig. 5.

Generally speaking, curved pipes are mainly characterised by the generation of vortices within the mainstream due to the imbalance between the pressure and centrifugal forces [21]; the vortices are generated as long as the centrifugal forces are present, otherwise they disappear as is the case in the straight pipes $\left(R_{c} \rightarrow \infty\right)$ [7]. The emergence of vortices produces significant and undesirable pressure drop in the fluid flow and the friction factor increases.

C-shaped pipes are alternating curvature pipes. The periodic changes in the orientation of bends lead to periodic reversal of centrifugal force, which means that there are regions where the centrifugal force must cancel out in order to change direction. With zero centrifugal force, vortices are no longer generated in these regions and the pressure will not be affected. The number of these regions increases as the periodicity of the $\mathrm{C}$-shaped pipes increases.

Thus, as the amplitude ratio $\beta$ increases, the number of periods per length (Frequency $\approx 1 / \lambda$ ) decreases as well as the number of regions without vortices: the more $\beta$ increases less pressure drop would increase, as shown in Figs. 6 and 7.

In the helically curved pipe, the direction of the centrifugal force with regard to the flow direction remains unchanged and the flow feature is unperturbed; the vortices should be continuously produced along the helical coil, as seen in Fig. $8 a$ and b, and the pressure should be continually affected and friction factor increases. In contrast to the coil pipes as explained previously, the C-shaped pipes are characterised by continuous periodic perturbation of the flow (Fig. 8c, d). Thus, compared to the helical coil, there are regions where the centrifugal force becomes zero and vortices are not generated and where
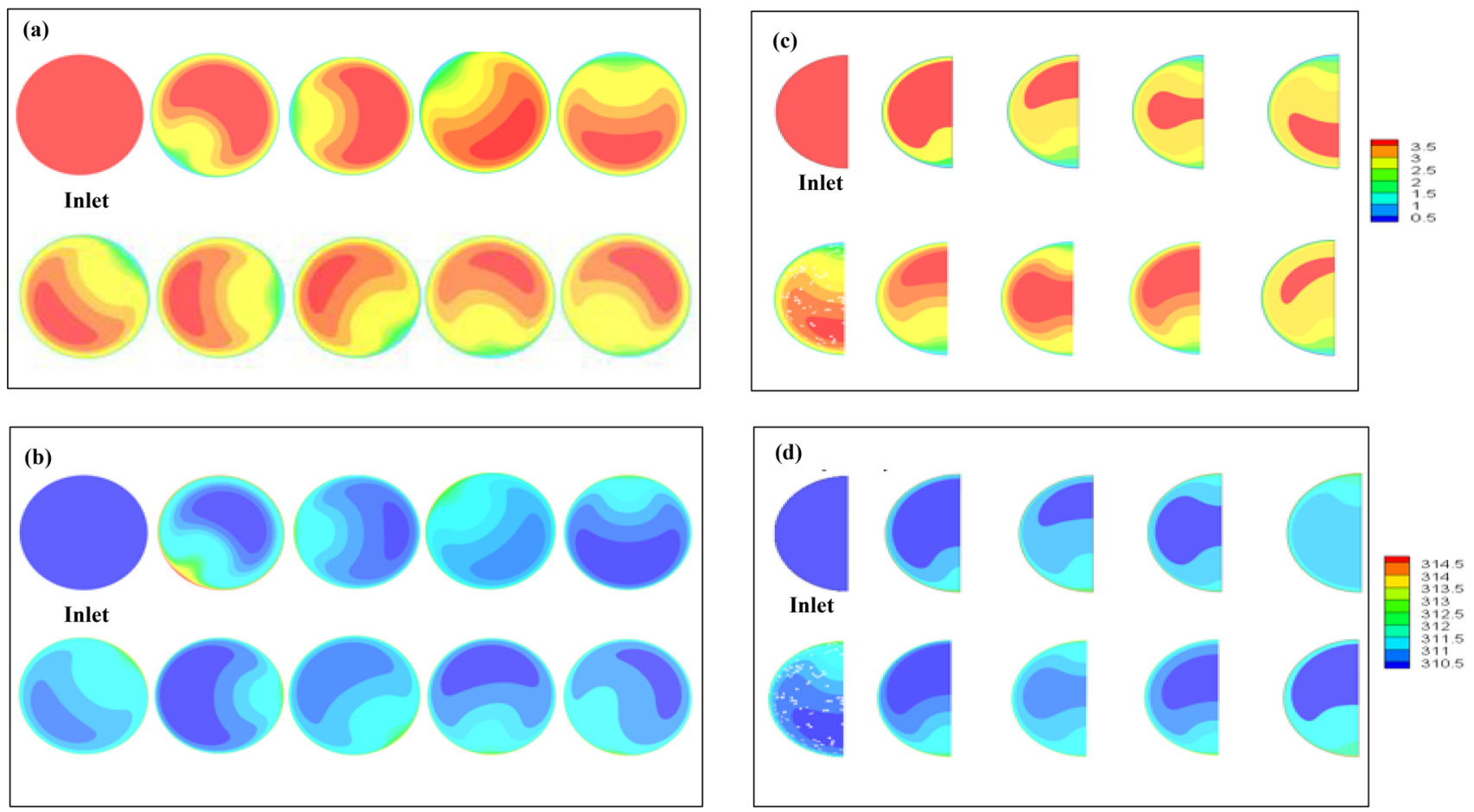

Fig. 8 The velocity $\left(\mathrm{m} \mathrm{s}^{-1}\right)$ and temperature $(\mathrm{K})$ distributions selected planes for $\mathrm{Re}=35353$. Along the helically coiled pipe, respectively (a) and (b). Along the C-shaped pipe $(\beta=1)$, respectively (c) and (d)

\section{SN Applied Sciences}


the pressure should not be affected; the friction factor $C_{f}$ for $C$-shaped pipes will be less important than the coils, as seen in Figs. 4 and 7.

Abou-Arab et al. [17] developed correlations for sinusoidal tubes, but these correlations are not practical for the C-shaped pipes, as seen in Fig. 7, as well as the correlation by Yang et al. [22] not discussed in this paper.

\section{Conclusions and perspectives}

The main conclusion of this study is that over the Reynolds number range $2 \times 10^{4}-5 \times 10^{4}$ the prediction of the internal friction factor $C_{f}$ in $C$-shaped pipes cannot be obtained using correlations established previously for helically pipes and would require further investigation to get to a convincing conclusion.

Unlike helical coils, the friction ratio $C_{f}$ of $C$-shaped pipe appears to be affected by ratios other than the curvature ratio as highlighted by the introduction of the amplitude ratio $\beta$. Within the range, $0.2 \leq \beta \leq 1$ the friction factor $C_{f}$ decreases as $\beta$ decreases.

In addition, the present subject is under ongoing investigations to:

- Study the effect of various parameters on heat transfer and its relationship to pressure drop;

- Study experimentally the thermodynamic behaviour of inside flows;

- Extend the study for more and various curved configurations.

\section{Compliance with ethical standards}

Conflict of interest The authors declare that they have no conflict of interest.

\section{References}

1. Hart J, Ellenberger J, Hamersma PJ (1988) Single and two-phase flow through helically coiled tubes. Chem Eng Sci 43(4):775-783

2. Guo L, Feng Z, Chen X (2001) An experimental investigation of the frictional pressure drop of steam-water two-phase flow in helical coils. Int J Heat Mass Transf 44:2601-2610

3. Mori Y, Nakayama W (1967) Study on forced convective heat transfer in curved pipes. Int J Heat Mass Transf 10(1):37-59; Part II 1967, Int J Heat Mass Transf 10(5):681-695, Part III 1967

4. Vashisth S, Kumar V, Nigam KDP (2008) A review on the potential applications of curved geometries in process industry". Ind Eng Chem Res 47(10):3291-3337
5. Demagh Y, Bordja I, Kabar Y, Benmoussa H (2015) A design method of an S-curved parabolic trough collector absorber with a three dimensional heat flux density distribution. Sol Energy 122:873-884

6. Demagh Y, Kabar Y, Bordja L, Noui S (2016) The 3D heat flux density distribution on a novel parabolic trough wavy absorber. In: AIP conference proceedings, vol 1734. https://doi. org/10.1063/1.4949151

7. Bitam E, Demagh Y, Hachicha AA, Benmoussa H, Kabar Y (2018) Numerical investigation of a novel sinusoidal tube receiver for parabolic trough technology. Appl Energy 218:494-510

8. Li LJ, Lin CX, Ebadian MA (1998) Turbulent mixed convective heat transfer in the entrance region of a curved pipe with uniform wall-temperature. Int J Heat Mass Transf 41:3793-3805

9. Jayakumar JS, Mahajani SM, Mandal JC, lyer KN, Vijayan PK (2010) CFD analysis of single-phase flows inside helically coiled tubes. Comput Chem Eng 34:430-446

10. Yang G, Ebadian MA (1996) Turbulent forced convection in a helicoidal pipe with substantial pitch". Int J Heat Mass Transf 39:2015-2022

11. Pawar SS, Sunnapwar VK (2014) Experimental and CFD investigation of convective heat transfer in helically coiled tube heat exchangers. Chem Eng Res Des 92:2294-2312

12. Di Piazza MC (2010) Numerical prediction of turbulent flow and heat transfer in helically coiled pipes. Int J Therm Sci 49:653-663

13. Menter FR (1994) Two-equation eddy-viscosity turbulence models for engineering applications. AIAA J 32:269-289. https://doi. org/10.2514/3.12149

14. Gnielinski V (1986) Heat transfer and pressure drop in helically coiled tubes. In: Proceedings of the eighth international heat transfer conference, vol 6. Tayler and Francis, Washington, DC, pp 2847-2854

15. Incropera FP, Dewitt DP, Bergman TL, Lavine AS (2007) Fundamentals of heat and mass transfer, 6 th edn. Wiley, Hoboken, $p$ 997

16. Ito $\mathrm{H}$ (1959) Friction factors for turbulent flow in curved pipes. J Basic Eng Trans ASME 81(2):123-132

17. Abou-Arab TW, Aldoss TK, Mansour A (1991) Pressure drop in alternating curved tubes. Appl Sci Res 48:1-9

18. Castiglia F, Chiovaro P, Ciofalo M, Di Liberto M, Di Maio PA, Di Piazza I, Giardina M, Mascari F, Morana G, Vella G (2010) Modelling flow and heat transfer in helically coiled pipes. Part 3: assessment of turbulence models, parametrical study and proposed correlations for fully turbulent flow in the case of zero pitch. Report RdS/2010/78

19. Vester AK, Örlü R, Alfredsson PH (2016) Turbulent flows in curved pipes: recent advances in experiments and simulations. Appl Mech Rev 68(5):050802

20. Di Liberto $M$, Ciofalo $M$ (2013) “A study of turbulent heat transfer in curved pipes by numerical simulation. Int J Heat Mass Transf 59:112-125

21. Eustic J (1911) Experiments of streamline motion in curved pipes. Proc R Soc Lond A85:1 19-131

22. Yang R, Chiang FP (2002) An experimental heat transfer study for periodically varying curvature curved-pipe. Int J Heat Mass Transf 45:3199-3204

Publisher's Note Springer Nature remains neutral with regard to jurisdictional claims in published maps and institutional affiliations. 